\title{
Effective Temperature Scales of Red Giant Stars
}

David R. Alexander, Jason W. Ferguson

Dept. of Physics, Wichita State University, Wichita, KS 67260-0032, $U S A$

Robert F. Wing

Astronomy Dept., Ohio State University, Columbus, OH 43210, USA

Hollis R. Johnson

Dept. of Astronomy, Indiana University, Bloomington, IN 47405, USA

Peter H. Hauschildt

Dept. of Physics $\mathcal{B}$ Astronomy, University of Georgia, Athens, GA 30602-2451, USA

France Allard ${ }^{1}$

Dept. of Physics, Wichita State University, Wichita, KS 67260-0032, USA

\begin{abstract}
.
We have completed a grid of spherically symmetric AGB star atmospheres using the state of the art spectral synthesis code PHOENIX. Models are constructed for stars with masses of $1 \mathrm{M}_{\odot}$ and $1.5 \mathrm{M}_{\odot}$, spanning the range 10 to $3300 \mathrm{~L}_{\odot}$ in luminosity and 2500 to $5200 \mathrm{~K}$ in effective temperature. We find that grains of $\mathrm{Al}_{2} \mathrm{O}_{3}$ and $\mathrm{CaTiO}_{3}$ among other species form in atmospheres cooler than $\mathrm{T}_{\text {eff }}=3000 \mathrm{~K}$. In the coolest models the grains cause a weakening of the TiO absorption features in the red and near infrared of up to $30 \%$ through both a depression of the continuum and a depletion of the $\mathrm{TiO}$ number abundance. We use spectrophotometric observations from a number of catalogs to determine effective temperature - spectral class and effective temperature - color relationships. We also compare synthetic colors calculated from our models with observations of $M$ giants on Wing's 8-color narrow-band system of classification photometry.
\end{abstract}

\footnotetext{
${ }^{1}$ Now at CRAL, Ecole Normale Superieure, Lyon, 69364 France Cedex 07
} 


\section{Introduction}

A great deal can be learned about AGB stars from accurate models of their photospheres. A comparison of the emergent spectra predicted by the models with flux-calibrated spectral observations makes possible the determination of the effective temperature, surface gravity, radius, luminosity, and surface chemical composition of individual stars. Because the photosphere must be the point of origin of the winds which emanate from these stars, an understanding of the thermal structure of the photosphere is fundamental to an understanding of the winds. The input physics and radiative transfer included in detailed calculations of model photospheres are required for an improved understanding of the hydrodynamic effects caused by stellar pulsations. Significant progress has been made in this field in recent years (see Gustafsson \& Jørgensen 1994, for a recent review of the field) as a result of the inclusion of more realistic physical assumptions in the models, made possible by the twin developments of improved input physics and the availability of ever faster computers and algorithms.

\section{Model photospheres: computational methods and input physics}

The models presented here have been computed with the multi-purpose stellar atmosphere code PHOENIX (Hauschildt, Allard \& Baron 1999; Hauschildt \& Baron 1998). This program can compute models in either plane-parallel or spherical geometry, with a fully self-consistent treatment of steady expansion, in either LTE or NLTE, and with incident illumination included. While many of these program characteristics will be important for a full understanding of the AGB phenomenon, for this report we consider only models which are in hydrostatic equilibrium, in LTE, computed in spherical geometry, and fully converged for energy conservation. It is interesting to note that a comparison of plane-parallel and spherical models shows that the flux of a $4200 \mathrm{~K}$ spherical model is depressed by about $15 \%$ in the violet compared to a plane-parallel model. At $3200 \mathrm{~K}$, the differences between spherical and plane-parallel models over the entire visual spectrum reach $40 \%$ (this effect is also seen in dwarf OB stars; Aufdenberg, Hauschildt \& Shore 1998). Because the plane-parallel models have more flux at short wavelengths than the spherical models, comparisons of plane-parallel models to observed spectra in the visual will result in an underestimation of the effective temperature, with a rapidly increasing effect for later spectral types.

The molecular opacities in all of our models are computed with the direct opacity sampling technique, with monochromatic opacities computed at 20000 wavelengths. Our spectral line database now includes more than 400 million molecular and atomic lines, including $\mathrm{H}_{2} \mathrm{O}, \mathrm{TiO}, \mathrm{CO}, \mathrm{CN}, \mathrm{HCN}$, and $\mathrm{C}_{2} \mathrm{H}_{2}$. The abundance of each chemical species is computed with an equation of state which assumes thermodynamic equilibrium exists. More than 850 molecules and 600 solids and liquids can be included in a fully self-consistent calculation. The equation of state program is based upon the work of Allard (Allard \& Hauschildt 1995; Alexander et al. 1997) and solves the equations of mass action for each species with full coupling between the gas phase and condensed species. 


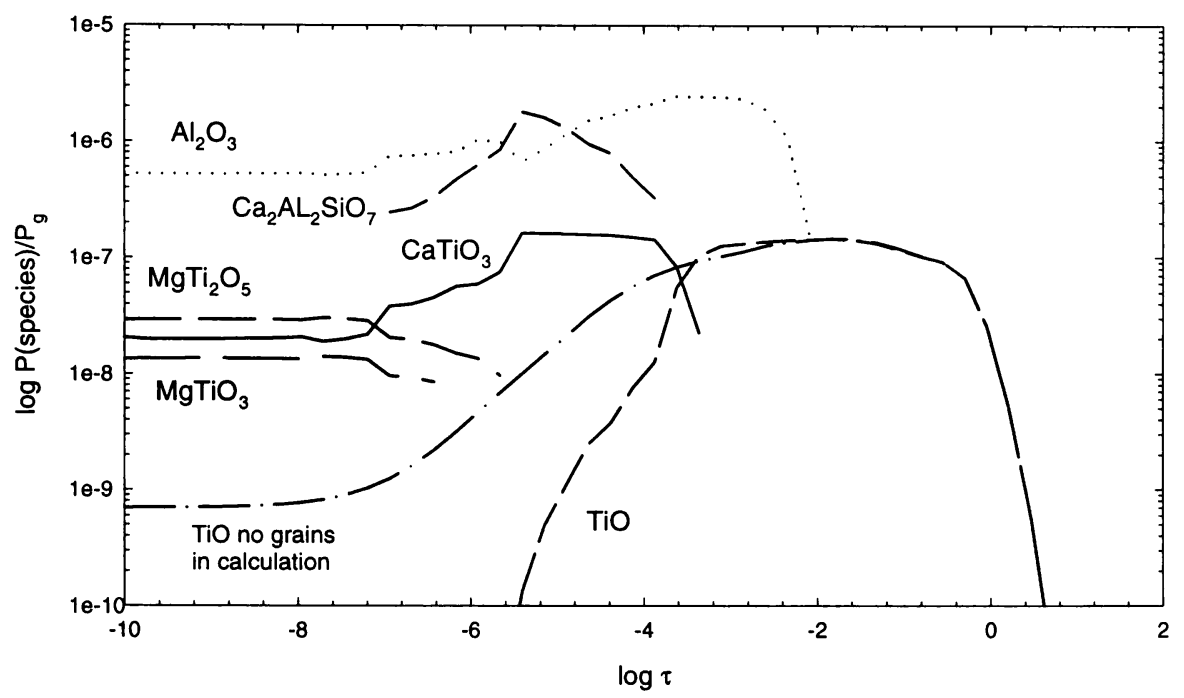

Figure 1. The fictitious partial pressures of condensed species in the photosphere of a $1.0 \mathrm{M}_{\odot}$ model with $\mathrm{T}_{\text {eff }}=2500 \mathrm{~K}$ and $\log g=-0.5$ are plotted versus the optical depth at $1.2 \mu \mathrm{m}$.

The opacity of solid grains is included with a Mie theory calculation for six different grain species, including two magnesium silicates, iron, corundum, and two carbon species. All grains are assumed to have the Mathis et al. (1977) power law size distribution determined from observations of the interstellar medium, which reasonably approximates the size distribution found by Dominik et al. (1993) in their calculation of dust formation in an M star wind.

\section{Model photospheres: comparison of the grid with observations}

For this report we have constructed a grid of 196 model photospheres which span the $\mathrm{K}$ and $\mathrm{M}$ spectral classes of red giant stars. All of these models have a solar composition, except that nitrogen has been enhanced by a factor of 2.5 and the carbon abundance has been reduced by a factor of 1.5 to match the abundances found by Smith \& Lambert (1990) for $18 \mathrm{M}$ giants. Future calculations will explore the effect of different $\mathrm{C} / \mathrm{O}$ ratios and metallicities and will expand the grid to higher luminosities and masses.

Our results lead to the discovery that grains condense in the photospheres of many of the models we compute. All models cooler than $\mathrm{T}_{\text {eff }}=3000 \mathrm{~K}$ with luminosities greater than $500 \mathrm{~L} \odot$ show grain condensation. While Tsuji (1998) has reported the existence of carbon grains in the atmospheres of some carbon stars, this is the first calculation which shows condensation in the photospheres of oxygen-rich giant stars. Fig. 1 shows the fictitious partial pressure of condensed species in one of our coolest models, which corresponds roughly to the M10 composite spectrum presented by Fluks et al. (1994). The first species to 
condense, at a temperature around $\sim 1530 \mathrm{~K}$, is $\mathrm{Al}_{2} \mathrm{O}_{3}$ at an optical depth of about 0.01 . When $\mathrm{CaTiO}_{3}$ condenses at an optical depth of $10^{-3}$, the important gas phase absorber $\mathrm{TiO}$ virtually disappears from the model. The net effect of grains on the spectrum of this model is to slightly depress the continuum, due to the enhanced continuous opacity of the grains, and to significantly weaken the $\mathrm{TiO}$ features in the visual. The $\mathrm{TiO}$ bands at $0.59 \mu \mathrm{m}$ and $0.62 \mu \mathrm{m}$ are reduced by approximately $50 \%$ in strength compared to a model computed without grain condensation. Since $\mathrm{TiO}$ band strengths are the standard indicator of spectral type, this result may have profound effects on the comparison of spectral type, color, and effective temperature. Among the species which condense in this model, optical constants are available only for $\mathrm{Al}_{2} \mathrm{O}_{3}$. A proper interpretation of late type spectra will require optical constants for the two calcium species which condense at relatively high temperatures.

The models in the grid have been fit to observations of stars taken from eleven published catalogs, which contain more than 300 energy-calibrated spectra of $\mathrm{K}, \mathrm{M}, \mathrm{S}$, and $\mathrm{C}$ stars. An automated procedure computes the root-meansquare ( $\mathrm{rms}$ ) deviation between a given observation and synthetic spectra for all the models in the grid smoothed to the same resolution as the observation. A plot of these rms deviations versus effective temperature usually shows a well defined minimum which accurately determines the effective temperature of the star. However, surface gravity is poorly determined by this technique because of its limited influence on the emergent spectrum. An example of the fit for the composite M3 spectrum of Fluks et al. (1994) is shown in Fig. 2. Stars with significantly different compositions or other peculiarities are easily identifiable from the large value of the minimum rms deviation. A direct comparison of the otserved and computed spectra is used to confirm the appropriateness of the fit.

The models have also been compared to observations of $50 \mathrm{M}$ giants obtained on the 8-color narrow band filter system of MacConnell, Wing \& Costa (1992). Fig. 3 (a) shows that the observations of 5 M2 giants are well matched between models with effective temperatures of $3700 \mathrm{~K}$ and $3800 \mathrm{~K}$, suggesting a range in temperatures for these stars of about $100 \mathrm{~K}$. The $\mathrm{TiO}$ indices and color temperatures computed for the models agree well with the observations, as seen in Fig. 3 (b), for a wide range of stars from K0 to M3.

Fig. 4 shows the effective temperature scale determined from a comparison of these models with three sets of observations. Variations in the effective temperature determined for stars of a given spectral class from the Burnashev (1982) catalog may represent differences in the properties of stars classified with the same spectral class, caused for example by differences in chemical composition. These effects are significantly reduced in the $\mathrm{T}_{\text {eff }}-(V-K)$ diagram. Differences in the effective temperature scale determined for the composite spectra of Fluks et al. (1994) and Silva \& Cornell (1992) are more likely the result of the finite model grid used.

\section{Conclusions}

We present here the results of the computation of a new grid of model photospheres for oxygen-rich red giant stars. All models have been computed in spherical geometry, with hydrostatic equilibrium, LTE, energy conservation, and 

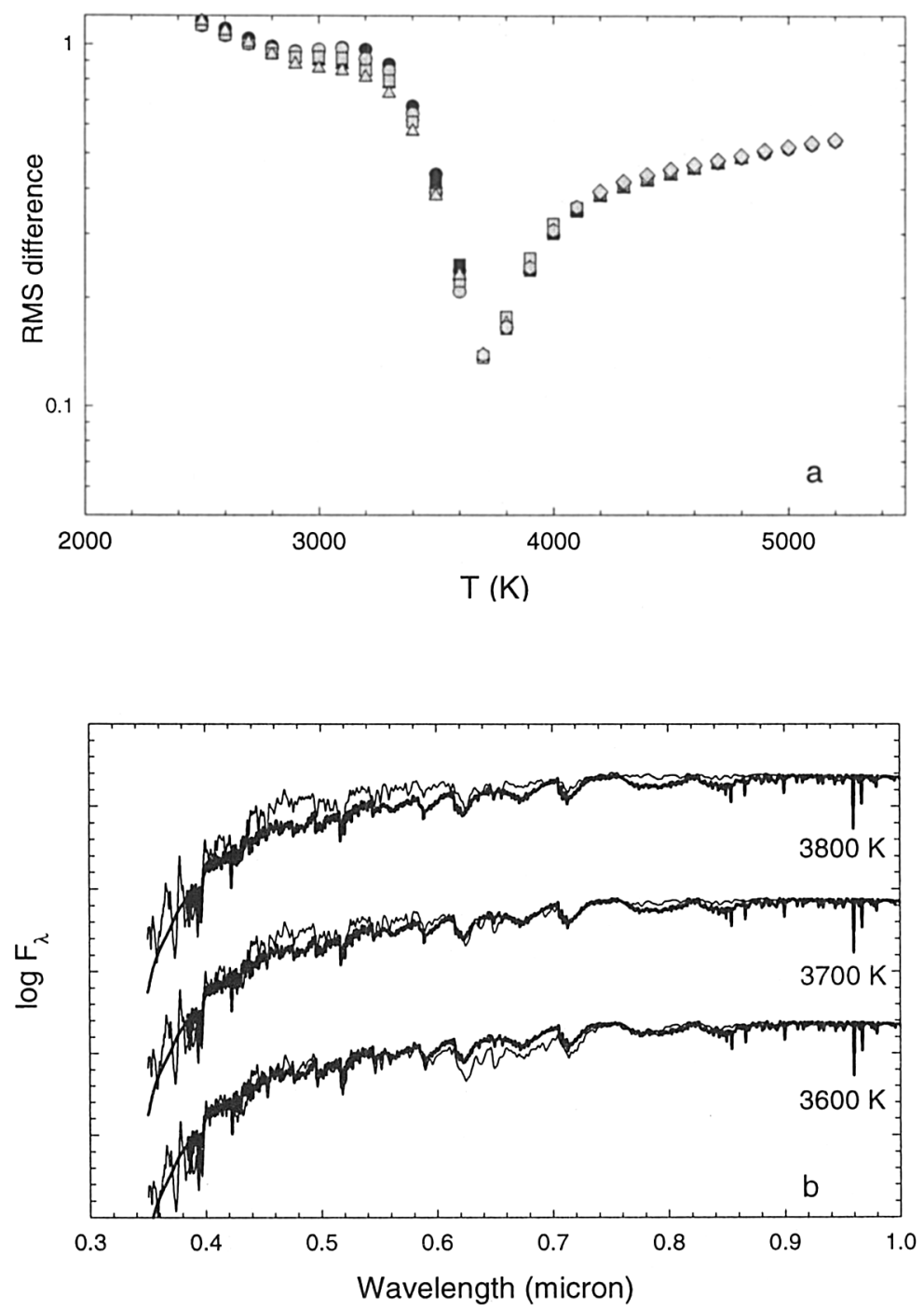

Figure 2. (a) The rms difference $\left(\chi^{2}\right)$ between the synthetic spectrum of an M3 giant observed by Fluks et al. (1994) and the models in the grid is plotted. Different symbols refer to models with differing $\mathrm{L}_{*}$ and $\mathrm{M}_{*}$. The minimum at $3700 \mathrm{~K}$ represents the best fitting models. Differences in $\log g$ are not well determined by this method. (b) The observed composite spectrum for M3 giants from Fluks et al. (1994) is plotted as the heavy line, along with models of three different effective temperatures. Differences caused by the temperature differences are obvious, although a human observer might have difficulty determining which model best fits the spectrum. 

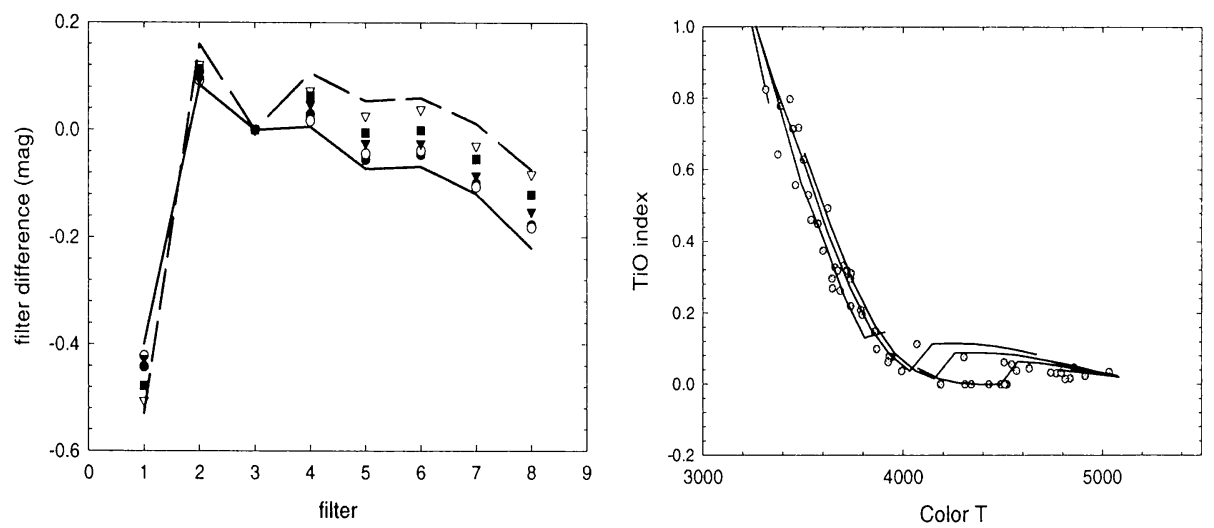

Figure 3. (a) Wing 8-color narrow band filter observations of 5 M2 standard giants are compared to synthetic colors computed from the models. The solid line are colors from a $3800 \mathrm{~K}$ model, and the dashed from a $3700 \mathrm{~K}$ model. All colors have been normalized to Wing filter 3 . (b) The $\mathrm{TiO}$ index and color temperature computed from the 8-color Wing observations of $50 \mathrm{~K} \& \mathrm{M}$ giants are compared to the values computed from synthetic colors of the models. From left to right, successive curves represent models of progressively higher luminosity from the grid.

chemical equilibrium including both gas phases and condensation of solids and liquids. All models cooler than $\mathrm{T}_{\text {eff }}=3000 \mathrm{~K}$ show grain condensation (including $\mathrm{Al}_{2} \mathrm{O}_{3}$ and $\mathrm{CaTiO}_{3}$ ) in the photosphere. This phenomenon profoundly affects the $\mathrm{TiO}$ band strength in the coolest models. The emergent spectra computed for these models have been compared to observations from a wide range of sources. The effective temperature scale determined from these comparisons agrees well with that determined from angular diameter observations (Ridgway et al. 1980). Future work will explore the effect of grain condensation, NLTE effects for atoms and the more abundant molecules, and the effects of changing the chemical composition.

Acknowledgments. Low Temperature Astrophysics research at Wichita State University is supported by NASA grants NCC5-168 and NAG5-3435. Some of the models reported here were computed from a generous allocation of computer time at the Center for Scientific Supercomputing at Kansas State University, supported by NSF grant CDA-9724289. PHH acknowledges support by NSF grant AST-9720704, NASA ATP grant NAG 5-3018 and LTSA grant NAG 5-3619 to the University of Georgia.

\section{References}

Allard F., Hauschildt P.H., 1995, ApJ 445, 433

Alexander D.R., Allard F., Tamani A., Hauschildt P.H., 1997, Ap\&SS 251, 171 

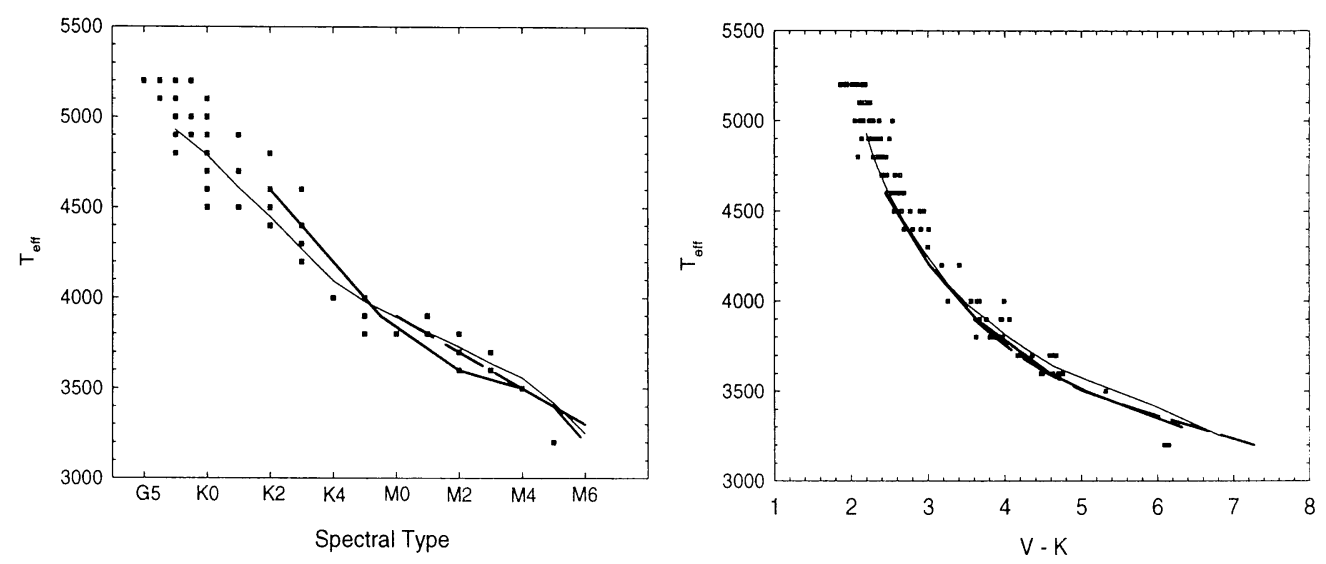

Figure 4. The effective temperature scale determined by fitting model spectra to observations from three catalogs as a function of (a) spectral class and (b) $V-K$ color. The square symbols are the Burnashev (1982) catalog, the thick solid line from Silva \& Cornell (1992), and the dashed line from Fluks et al. (1994). The effective temperature scale determined by Ridgway et al. (1980) from angular diameter measurements is also given (thin solid line).

Aufdenberg J.P., Hauschildt P.H., Shore S.N., 1998, ApJ 498, 837

Burnashev V.I., 1982, Izv. Krymskoi Astro. Obs. 65, 94

Dominik C., Sedlmayr E., Gail H.-P., 1993, A\&A 277, 578

Fluks M.A., Plez B., The P.S., De Winter D., Westerlund B.E., Steenman H.C., 1994, A\&AS 105, 311

Hauschildt P.H., Allard F., Baron E., 1999, ApJ 512, 377

Hauschildt P.H., Baron E., 1998, J. Comp. App. Math., in press

Gustafsson B., Jørgensen U.G., 1994, A\&A Rev. 6, 19

MacConnell D.J., Wing R.F., Costa E., 1992, AJ 104, 821

Mathis J.S., Rumpl W., Nordsieck K.H., 1977, ApJ 217,425

Ridgway S.T., Joyce R.R., White N.M., Wing R.F., 1980, ApJ 235, 126

Silva D.R., Cornell M.E., 1992, ApJ 81, 865

Smith V.S., Lambert D.L., 1990, ApJS 72, 387

Tsuji T., 1998, in The Carbon Star Phenomenon, ed. R.F. Wing, IAU Symp. 177, Kluwer Academic Publishers, in press 